\title{
BMJ Open Risk assessment of patient factors and medications for drug-related problems from a prospective longitudinal study of newborns admitted to a neonatal intensive care unit in Brazil
}

\author{
Ramon D Leopoldino, ${ }^{1}$ Marco T Santos, ${ }^{2}$ Tatiana X Costa, ${ }^{2}$ Rand R Martins, ${ }^{1}$ \\ António G Oliveira ${ }^{1}$
}

To cite: Leopoldino RD, Santos MT, Costa TX, et al. Risk assessment of patient factors and medications for drug-related problems from a prospective longitudinal study of newborns admitted to a neonatal intensive care unit in Brazil. BMJ Open 2019;9:e024377. doi:10.1136/ bmjopen-2018-024377

- Prepublication history and additional material for this paper are available online. To view these files, please visit the journal online (http://dx.doi org/10.1136/bmjopen-2018024377).

Received 23 May 2018 Revised 18 March 2019 Accepted 20 June 2019

Check for updates

(C) Author(s) (or their employer(s)) 2019. Re-use permitted under CC BY-NC. No commercial re-use. See rights and permissions. Published by BMJ.

${ }^{1}$ Department of Pharmacy, Universidade Federal do Rio Grande do Norte, Natal, Brazil ${ }^{2}$ Maternidade Escola Januário Cicco, Universidade Federal do Rio Grande do Norte, Natal, Brazil

Correspondence to Mr Ramon D Leopoldino; ramon.weyler@gmail.com

\section{ABSTRACT}

Objective To identify patient factors and medications associated with the occurrence of drug-related problems (DRPs) in neonates admitted to neonatal intensive care units (NICUs).

Design Prospective, longitudinal study.

Setting NICU of a teaching hospital in Brazil.

Participants Data were collected from the records of the clinical pharmacy service of all neonates admitted between April 2014 and January 2017, excluding neonates with length of stay in the NICU $<24$ hours or without prescribed drugs.

Primary outcome measures 0ccurrence of one or more DRP (conditions interfering in the patient's pharmacotherapy with potential undesired clinical outcomes).

Results The study observed 600 neonates who had a median length of stay in the NICU of 13 days (range 2-278 days). DRPs were identified in most neonates $(60.5 \%)$. In a multivariate logistic regression model, the factors independently associated with DRP were gestational age (adjusted OR (AOR) 0.85, 95\% Cl 0.81 to 0.89 ), $5 \mathrm{~min}$ Apgar $<7$ (AOR 1.74, 95\% Cl 1.00 to 3.13), neurological disease (AOR 2.49, 95\% Cl 1.09 to 5.69), renal disease (AOR $5.75,95 \% \mathrm{Cl} 1.85$ to 17.8) and cardiac disease (AOR $2.36,95 \% \mathrm{Cl} 1.31$ to 4.24$)$. The medications with greater risk for DRP were amphotericin B (AOR 4.80), meropenem (AOR 4.09), alprostadil (AOR 3.38), vancomycin (AOR 3.34), ciprofloxacin (AOR 3.03), gentamicin (AOR 2.43), cefepime (AOR 1.88), amikacin (AOR 1.82) and omeprazole (AOR 1.66). These medicines represented one-third of all prescribed drugs.

Conclusions Gestational age, 5 min Apgar $<7$, and neurological, cardiac and renal diseases are risk factors for DRP in NICUs. Alprostadil, omeprazole and several antiinfectives were associated with greater risk of DRP.

\section{INTRODUCTION}

The intensive care unit (ICU) is a complex environment, characterised by polypharmacy, transfusions and frequent surgical procedures. ${ }^{1}$ Neonatal intensive care units
Strengths and limitations of this study

- This is the first study identifying patient variables and drugs associated with the occurrence of drug-related problems (DRPs), exclusively in neonatal intensive care unit patients.

- The validity of the results is strengthened by the large cohort size, the prospective data collection, the longitudinal design, the in situ evaluation of DRP and the adoption of a well-known DRP classification system.

- However, the data were obtained from a single institution, which may somehow impair the generalisation of our findings.

- It is also possible that administration errors have been underestimated by recording failures.

(NICUs) may pose additional hazards to patient because of the frequent usage of off-label and unlicensed medicines and of continued need for decimal dilutions of intravenous medicines prior to delivery. ${ }^{23}$ In addition, because of their physiological immaturity and rapid growth, neonates exhibit large interindividual variability in drug metabolism and excretion. ${ }^{2}$ Such characteristics may predispose neonates to drug-related problems (DRPs).

DRPs are events or circumstances arising from the patient's pharmacotherapy that may actually or potentially interfere with health outcomes. ${ }^{4}$ Those events include errors in the drug therapy processes (prescription, dispensation and administration) and adverse drug events (any untoward event related to medication that results in harm to the patient) ${ }^{5-7}$

In paediatric wards, DRP occur in about half of the patients, and most are preventable. ${ }^{8}$ 
However, there is very little information on DRP in children in NICUs, especially among neonates.

It is believed that DRPs are particularly frequent and serious in neonates. ${ }^{910}$ Neonates are very sensitive to dose variations because of their particular pharmacokinetics and pharmacodynamics, consequence of the lower drug metabolism and clearance, low levels of plasma proteins, high proportion of body water, and level of receptor expression and sensitivity. ${ }^{11}{ }^{12}$ Some authors have shown that harm involving medicines is common in NICUs, with incidence rates ranging from 10 to 20 cases per 1000 patient-days. ${ }^{13}{ }^{14}$ Such harm can lead to prolonged hospitalisation time and, in extreme cases, to the death of patients. It also generates an increase in hospital costs. ${ }^{13} 15$ Thus, the development of effective preventive strategies directed to DRP is of great relevance for the improvement of healthcare, and one step towards this goal is the identification of patients susceptible to DRP. ${ }^{16}$

Therefore, the purposes of this study were to identify risk factors for the occurrence of one or more DRP in NICU, to assess the risk associated with commonly used medications, and to describe the causes of DRP in the medications with greater risk of DRP in neonates.

\section{METHODS}

This was an observational, prospective, longitudinal study conducted from April 2014 to January 2017 in the 20-bed NICU of a teaching maternity hospital specialised in high-risk pregnancy. All neonates admitted to the NICU during the study period were prospectively evaluated for inclusion in the study. Inclusion criteria were a NICU stay longer than 24 hours and at least one prescribed drug. Neonates who were prescribed exclusively with electrolytes, parenteral nutrition, blood products, oxygen therapy, diagnostic agents, and vitamin and mineral supplements were excluded from the study, as these products were not considered as drugs.

In the absence of information in the literature on risk factors for DRP in neonates, the patient variables selected as candidates for assessment in a multivariate risk model were those that could be collected at NICU admission on every neonate and that reflect serious conditions that are usually associated with enhanced pharmacotherapy. The data collected from each neonate included sex, gestational age, birth weight, type of delivery (vaginal or caesarean), occurrence of premature rupture of membranes (PROM), $1 \mathrm{~min}$ and 5 min Apgar, a diagnosis of neurological, renal or cardiac disorder, and malformations. The Apgar is a score that evaluates the birth condition of newborns in the first and fifth minutes of life, with values below 7 being considered an ominous sign. ${ }^{17}$ In addition to those risk factors that may be predictors of DRP, patient variables representing the complexity of care (number of unique medications prescribed, number of different clinical problems and NICU length of stay in days) were also collected from each patient.
The study also wanted to identify medications that were associated with increased risk of DRP in neonates, and therefore all the medications prescribed to each neonate during the NICU stay were recorded.

The identification of DRP was actively performed on a daily basis by the NICU clinical pharmacy team (a chief pharmacist and four assistant pharmacists) through the analysis of medical charts, medication orders and nursing records, seeking entries that might indicate the occurrence of a DRP. The pharmacists involved in this research were permanent members of the clinical pharmacy team allocated to the NICU of our institution. The identification of DRP and their notification to the medical team are an important part of their routine work, and all were experienced in the detection of DRP. For each identified DRP, its causes were then classified according to the Pharmaceutical Care Network Europe system V.6. $2^{4}$ (see online supplementary file 1 ). This classification was carried out independently by two pharmacists (RDL and MS), supported by the Neofax textbook (Thomson Reuters, New York, USA), as well as the Micromedex (Truven Health Analytics, Michigan, USA) and UpToDate (Wolters Kluwer, Alphen aan den Rijn, the Netherlands) databases, which provided authoritative information on adverse drug reactions and drug-drug interactions. Whenever the two evaluators disagreed on the classification of the cause of a DRP, a third pharmacist (TC) was called in to break the tie.

\section{Statistical analysis}

The target sample size was set at 600 patients, a number that would afford $70 \%$ power to identify associations with an OR of 1.30 or greater for patient factors with a prevalence over $30 \% .{ }^{18}$ All variables are described by mean $\pm \mathrm{SD}$, median (range), or as absolute and relative frequency, as appropriate. For the identification of risk factors of DRP, an initial selection of patient variables at NICU admission (sex, gestational age, birth weight, type of delivery, occurrence of PROM, 1 min and 5 min Apgar, a diagnosis of neurological, renal or cardiac disorder, and malformations) were tested for association with the occurrence of one or more DRP with logistic regression. All variables were binary, except gestational age and birth weight which were continuous. The set of patient variables whose association with DRP was statistically significant at the 0.10 significance level in univariate logistic regression was analysed by stepwise backward multiple logistic regression, and those variables significant at the 0.05 level were retained in the final model. Variables collected only at discharge from the NICU (number of unique medications, length of stay and number of clinical problems) were analysed in a separate logistic model consisting of those three variables. Results of these analyses are presented as OR adjusted by the other variables in the model (AOR) and 95\% CI. The model was $\ln [p(\mathrm{DRP}=1) / p(\mathrm{DRP}=0)]=\beta_{0}+\beta_{i} x_{i}$, where $\beta_{0}$ is the regression constant, $\beta_{i}$ the partial regression coefficients and $x_{i}$ the independent variables. 
It was hypothesised that some medications could be singled out because they are associated with a significantly higher risk of DRP, through a combination of complex dosing and/or administration, and of frequency of use. Those drugs would be high-risk medications requiring close monitoring from the clinical pharmacy team. In the NICU setting, very often several medications are prescribed concurrently, sometimes simultaneously through the same intravenous line, and accounting for the interplay of all medications administered to a patient at a given day in a statistical model would be unmanageable. Therefore, the estimation of the risk of DRP associated with each medication was based on a simpler model, where the risk of DRP observed with a given medication was compared with the average risk observed with all other medications prescribed to this patient population, controlling for covariables. For this analysis, a set of multiple logistic regressions with each drug as independent variable and adjusted by the risk factors at NICU admission identified in the previous analysis were evaluated and, for those medications where a statistically significant association with the occurrence of one or more DRP was found at the 5\% significance level, results are presented as AOR of DRP with that medication to the average risk of all the other medications prescribed. The model was $\ln [p(\mathrm{DRP}=1) / p(\mathrm{DRP}=0)]=\beta_{0}+\beta_{1} x_{1}+\beta_{i} x_{i}$, where $\beta_{0}$ is the regression constant, $\beta$ the partial regression coefficients, $x_{1}$ is a binary variable coding for the medication, and $x_{i}$ the covariables. In the drugs identified in the previous analysis as high-risk medications, the respective causes of DRP are presented descriptively. The interaction of each of those high-risk medications with each risk factor previously identified was tested with multiple logistic regression, with significant interactions assumed at the $\mathrm{p}<0.10$ level. The model was $\ln [p(\mathrm{DRP}=1) / p(\mathrm{DRP}=0)]=\beta_{0}+\beta_{1} x_{1}+\beta_{i} x_{i}+\beta_{j} x_{1} x_{i}$, where $\beta_{0}$ is the regression constant, $\beta$ the partial regression coefficients, $x_{1}$ is a binary variable coding for the medication, $x_{i}$ the covariables and $\beta_{i} x_{1} x_{i}$ the interaction of the medication with each covariable. Statistical analysis was performed with Stata V.11.

\section{Patient and public involvement}

Patients were not involved in design or planning the study.

\section{RESULTS}

During the 34-month study period, a total of 627 newborns were admitted to the NICU. Of these, 15 newborns were excluded (13 because they had no drugs prescribed and 2 patients in whom the length of stay was less than 24 hours). Six hundred and twelve newborns remained eligible, but $12(1.96 \%)$ were excluded from the analysis because they had missing pharmacotherapy follow-up data. The analysis set of 600 newborns was observed for a total of 15836 NICU days, with a median of 13 days (range 2-278 days). The study population consisted of 265 girls $(45.1 \%)$ and the mean gestational age was $32.1 \pm 4.1$ weeks. On average,
Table 1 Demographic and clinical characteristics of the study population $(n=600)$

\begin{tabular}{ll}
\hline Characteristics & Value $^{*}$ \\
\hline Gestational age (weeks) & $32.1 \pm 4.1$ \\
\hline Female sex & $265(45.1)$ \\
\hline Birth weight (kg) & $1.80 \pm 0.88$ \\
\hline Length of stay (days) & $13(2-278)$ \\
\hline Vaginal delivery & $207(35.2)$ \\
\hline PROM & $162(31.5)$ \\
\hline 1 min Apgar $<7$ & $266(45.8)$ \\
\hline 5 min Apgar $<7$ & $76(12.9)$ \\
\hline Number of clinical conditions & $4.7 \pm 2.6$ \\
\hline Neurological disorders & $49(8.2)$ \\
\hline Renal disorders & $52(8.7)$ \\
\hline Cardiac disorders & $107(17.9)$ \\
\hline Malformations & $69(11.5)$ \\
\hline Number of medications used & $8.2 \pm 6.0$ \\
\hline DRP ( $\mathrm{n}=1115)$ & \\
\hline Patients with DRP & $363(60.5)$ \\
\hline Average number of DRP per patient & $1.9 \pm 2.6$ \\
\hline Death & $68(11.3)$ \\
\hline
\end{tabular}

*Values are mean \pm SD, median (range) or $n(\%)$. DRP, drug-related problem; PROM, premature rupture of membranes.

$8.2 \pm 6.0$ medicines were prescribed to each newborn during the NICU stay. A total of 1115 DRPs were identified, with a mean of $1.9 \pm 2.6 \mathrm{DRP}$ per patient. There were $237(39.5 \%)$ patients with no DRP, $132(22.0 \%)$ with one DRP, $71(11.8 \%)$ with two DRPs, and 160 (26.7\%) with three or more DRPs. Multiple DRPs in the same patient could occur concurrently or simultaneously. Sixty-eight neonates $(11.3 \%$ ) died during the study (table 1 ).

As shown in table 2, univariate logistic regression analysis identified eight patient variables at admission that were associated with DRP: lower gestational age, lower birth weight, vaginal delivery, $1 \mathrm{~min}$ and $5 \mathrm{~min}$ Apgar $<7$, neurological disorder, renal disorder and cardiovascular disorder. In the multivariate logistic regression model, five remained significant: lower gestational age (31.2 \pm 4.1 vs $33.5 \pm 3.7$ weeks, AOR 0.85, p<0.01), 5 min Apgar $<7$ (73.7\% vs 26.3\%, AOR 1.74, p $<0.01)$, neurological disorder $(75.5 \%$ vs $24.5 \%$, AOR $2.49, \mathrm{p}=0.03)$, renal disorder $(92.3 \%$ vs $7.7 \%$, AOR $5.75, \mathrm{p}<0.01)$ and cardiac disorder $(73.8 \%$ vs $26.2 \%$, AOR 2.36, $\mathrm{p}<0.01)$ were risk factors at admission for DRP. The $c$-statistic for the multivariate model with five variables was 0.72 .

DRPs were associated with increased length of stay (38.2 \pm 39.6 days vs $10.8 \pm 9.9$ days, AOR 1.04, $\mathrm{p}<0.001)$, number of prescribed drugs $(10.6 \pm 6.3$ vs $4.6 \pm 3.0$, AOR 1.22, $\mathrm{p}<0.001)$ and number of clinical problems $(5.57 \pm 2.86$ vs $3.39 \pm 1.51$, AOR $1.22, \mathrm{p}<0.001)$. There was 
Table 2 Factors associated with occurrence of drug-related problems in neonatal intensive care

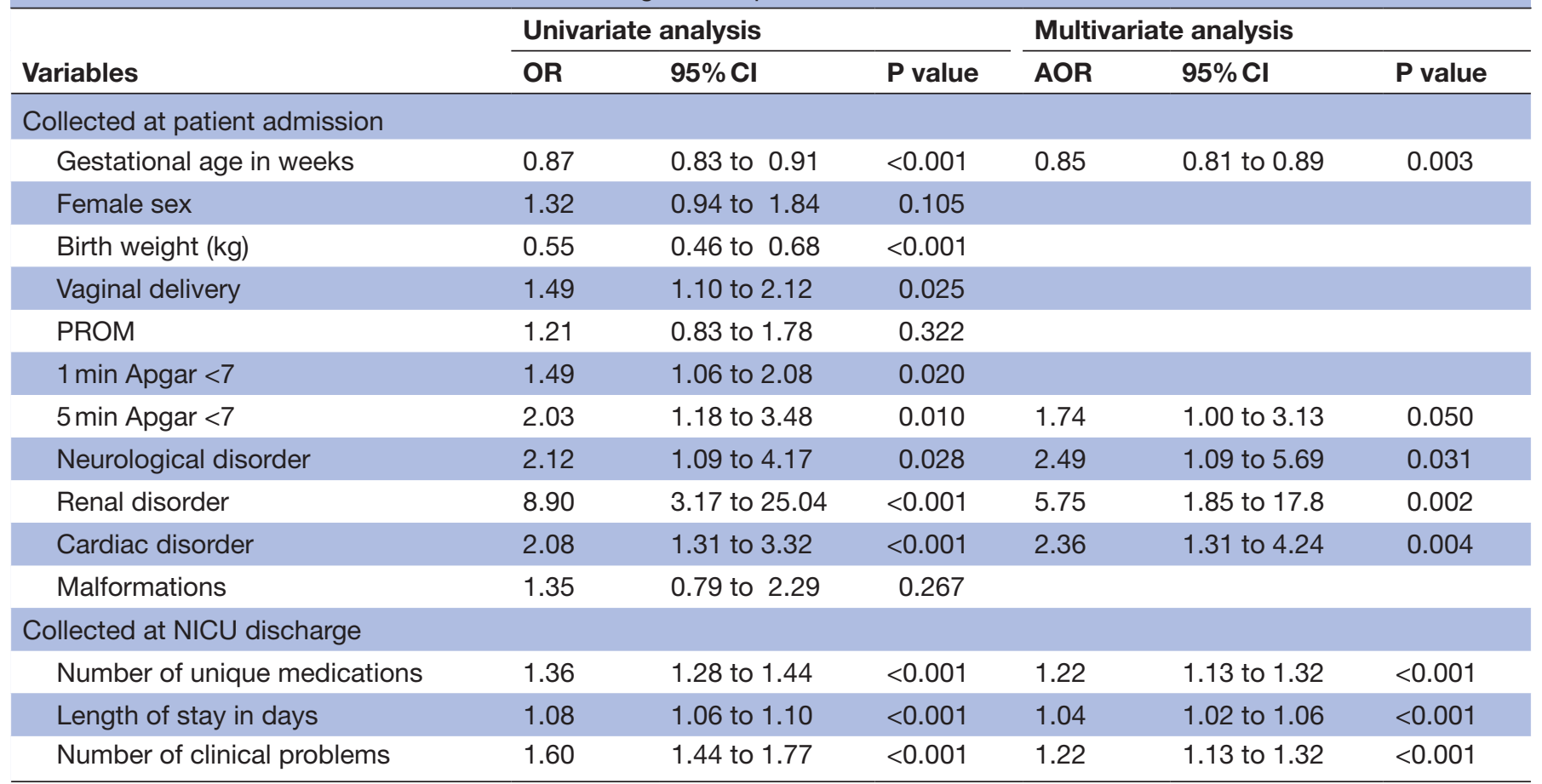

AOR, adjusted OR; NICU, neonatal intensive care unit; PROM, premature rupture of membranes.

no evidence of an association with a fatal outcome $(11.4 \%$ vs $11.3 \%, \mathrm{p}=0.702)$.

Table 3 shows the medicines with a statistically significantly increased risk of DRP compared with all the other prescribed medicines, adjusted for gestational age, $5 \mathrm{~min}$ Apgar score $<7$, neurological disorder, renal disorder and cardiac disorder. The medications, and their corresponding AORs, were amphotericin B (AOR 4.80), meropenem (AOR 4.09), alprostadil (AOR 3.38), vancomycin
(AOR 3.34), ciprofloxacin (AOR 3.03), gentamicin (AOR 2.43), cefepime (AOR 1.88), amikacin (AOR 1.82) and omeprazole (AOR 1.66). Related to increase in the occurrence of DRP, there were statistically significant interactions between renal disease and the prescription of amphotericin $(p=0.084)$ and of meropenem $(p=0.054)$, and between a 5 min Apgar score $<7$ and prescription of vancomycin $(\mathrm{p}=0.038)$.

Table 3 Estimates of the risk of DRP associated with several drugs administered in NICUs, distributed by cases of DRP and frequency of prescription

\begin{tabular}{|c|c|c|c|c|c|}
\hline \multirow[b]{2}{*}{ Medicines } & \multirow[b]{2}{*}{ Adjusted OR $(95 \% \mathrm{Cl})^{*}$} & \multicolumn{2}{|c|}{ Cases of DRP } & \multicolumn{2}{|c|}{ Frequency of prescriptions } \\
\hline & & $\mathbf{n}$ & $\%$ & $\mathbf{n}$ & $\%$ \\
\hline Amphotericin B & $4.80(1.49$ to 15.40$)$ & 46 & 3.7 & 48 & 1.0 \\
\hline Alprostadil & 3.38 (1.67 to 6.84$)$ & 16 & 1.3 & 33 & 0.7 \\
\hline Vancomycin & 3.34 (1.17 to 9.52$)$ & 97 & 7.7 & 100 & 2.0 \\
\hline Gentamicin & $2.43(1.00$ to 5.89$)$ & 211 & 16.9 & 518 & 10.5 \\
\hline Cefepime & $1.88(1.13$ to 3.13$)$ & 38 & 3.0 & 193 & 3.9 \\
\hline Amikacin & 1.82 (1.09 to 3.07$)$ & 73 & 5.8 & 181 & 3.7 \\
\hline Omeprazole & 1.66 (1.02 to 2.59$)$ & 28 & 2.2 & 146 & 3.0 \\
\hline Others & & 630 & 50.3 & 3522 & 71.6 \\
\hline
\end{tabular}

${ }^{*}$ OR adjusted for gestational age, $5 \mathrm{~min}$ Apgar $<7$, neurological disorder, renal disorder and cardiac disorder. The $p$ value for each medicine was $<0.05$.

DRP, drug-related problem; NICU, neonatal intensive care unit. 
Table 4 Type and frequency of the causes of DRP in medicines associated with high risk of DRP in neonatal intensive care

\begin{tabular}{|c|c|c|c|c|c|}
\hline \multirow[b]{2}{*}{ Medicines } & \multicolumn{5}{|l|}{ Causes of DRP* } \\
\hline & Drug selection & Dose selection & Drug use process & Logistics & Others $†$ \\
\hline Amphotericin B & & $7(15.2 \%)$ & $21(45.7 \%)$ & $11(23.9 \%)$ & $7(15.2 \%)$ \\
\hline Alprostadil & & $2(12.5 \%)$ & $6(37.5 \%)$ & & $8(50.0 \%)$ \\
\hline Vancomycin & $1(1.0 \%)$ & $28(28.9 \%)$ & $24(24.74 \%)$ & $40(41.24 \%)$ & $4(4.12 \%)$ \\
\hline Gentamicin & & $132(62.6 \%)$ & $73(34.6 \%)$ & $4(1.9 \%)$ & $2(0.9 \%)$ \\
\hline Cefepime & & $16(42.1 \%)$ & $10(26.32 \%)$ & $11(28.95 \%)$ & $1(2.63 \%)$ \\
\hline Amikacin & & 47 (64.4\%) & $14(19.2 \%)$ & 8 (10.9\%) & $4(5.5 \%)$ \\
\hline Omeprazole & & $1(3.57 \%)$ & $15(53.57 \%)$ & $12(42.86 \%)$ & \\
\hline
\end{tabular}

${ }^{*}$ Causes of DRP according to the Pharmaceutical Care Network Europe classification system V.6.2. ${ }^{4}$

†Others included drug form, treatment duration and other specific causes (eg, adverse reaction and wrong drug preparation technique). DRP, drug-related problem.

The frequency of prescription and the prevalence of DRP related to those medications are also displayed in table 3. These nine drugs represent $28.4 \%$ (1395/4917) of all medications prescribed in the NICU and accounted for $49.7 \%(622 / 1252)$ of problems involving medications. The most prescribed medicines in the group were gentamicin $(10.5 \%, 518)$ and meropenem $(3.1 \%, 152)$, and these drugs were also the most often involved in DRP (16.9\% (211) and 8.0\% (100), respectively).

As for the causes of DRP involving the nine medicines (table 4), dose selection was the most common cause for gentamicin $(62.6 \%)$, amikacin $(64.4 \%)$, meropenem $(38.0 \%)$, cefepime $(42.1 \%)$ and ciprofloxacin $(30.77 \%)$. DRPs involving omeprazole $(53.57 \%)$ and amphotericin B $(45.7 \%)$ were most often related to drug use process. Alprostadil was mainly involved in other causes such as wrong drug preparation technique $(18.75 \%)$ and suspected adverse reaction $(31.25 \%)$. Vancomycin was most often implicated in errors of prescription logistics $(41.24 \%)$.

\section{DISCUSSION}

In our study, we observed that neonates with low gestational age, low 5 min Apgar, neurological disorder, renal disorder and cardiac disorder are more likely to have DRP during their stay in NICU. An assessment of the risk of DRP was made for alprostadil, amikacin, amphotericin B, cefepime, ciprofloxacin, gentamicin, meropenem, omeprazole and vancomycin. Such medicines accounted for less than one-third of the drugs prescribed in the NICU, and were involved in half of DRPs, the majority being related to drug dose and to drug use.

Only a few studies have identified risk factors for the occurrence of DRP in hospitalised patients. Most of those studies were conducted in adult and paediatric wards for periods under 6 months and enrolled fewer than 400 patients. ${ }^{19-22}$ We performed a study in the NICU involving
600 neonates for a period of 3 years and presenting a set of different predictor variables. Comparisons with the results of other studies are therefore difficult. Even so, several risk factors related to DRP identified in our study, such as age and clinical problems (cardiac, neurological and renal disorders), were also observed in the works of Urbina et al, ${ }^{16}$ Peterson and Gustafsson, ${ }^{21}$ and Blix $e t a l,{ }^{23}$ although these studies were conducted in adult patients.

We also found that a low 5 min Apgar was associated with a higher risk of DRP. This predictor is a specific neonatology parameter that measures the condition of the newborn at birth. ${ }^{24}$ A low Apgar score usually represents a serious situation with the corresponding need for several therapeutic interventions which, in turn, increase the risk of DRP.

The detection of the clinical variables associated with DRP, as well as knowledge of the risk of DRP associated with each medication, represents a first step for the development of preventive strategies for enhanced patient safety and improvements in the process of care. Blix et $a l^{23}$ were the first authors to present risk estimates for drugs, while other papers ${ }^{8-22} 2526$ have only described drugs involved in DRP. We were able to quantify the risk of DRP for a set of drugs that are involved in half of all DRPs, namely alprostadil, omeprazole and several antimicrobials (amikacin, cefepime, ciprofloxacin, gentamicin, meropenem, amphotericin B and vancomycin). Pawluk et $a l^{25}$ and Stavroudis $e t a l^{26}$ claimed that the risk of DRP associated with a medicine is directly related to the frequency of prescription. However, our results show that the medicines with greater odds of DRP (vancomycin and amphotericin B) were not the most prescribed. These results suggest that the risk of DRP is primarily associated with the chemical and pharmacological properties of a drug, therefore strongly related to the level of difficulty on setting the appropriate dose and on the drug's potential for adverse reactions, interactions and incompatibilities. 
Inappropriate dose selection was the most common cause of DRP for aminoglycosides, cefepime and meropenem. In neonates, the adjustment of dose and regimen of antibiotics is extremely complex, the main reason for this being the rapid change in weight during the first days of life, as well as significant heterogeneity in the maturation of organs and systems across newborns. ${ }^{12} 27$ The lower than the recommended doses of those medicines administered in this study were often due to a delay in the adjustment of the medication dose to the rapid weight gain of the neonate.

We observed that amphotericin B, ciprofloxacin and omeprazole were associated with inappropriate process of drug use, specifically with drug administration error, with drug incompatibility being the most frequent cause. Neonates have a high risk of exposure to drug incompatibilities because of the limited number of intravenous accesses, often leading to simultaneous administration of incompatible drugs through the same intravenous line. In addition, the requirements for delivery of drugs in this population, such as dilutions and reduced infusion rates, can lead to incompatibilities because of high concentrations and longer time of contact between incompatible medicines. ${ }^{28}$ Such problems may be implicated in therapeutic failures due to drug degradation and even to thromboembolic complications, including cases of deaths, due to the precipitate formed reaching the bloodstream. ${ }^{29-31}$

Another medicine that had potential incompatibilities as the main cause of DRP was alprostadil. However, this medicine stands out for the significant percentage of cases of suspected adverse reactions. Fever, leucocytosis and dyspnoea are reactions commonly observed in neonates soon after the administration of alprostadil. ${ }^{32}$ Because of these reactions and complications, this medication is for intensive therapy only.

The most common cause of vancomycin-related problems was errors of prescription logistics. These errors are characterised by the lack of important information in the prescription for the safe administration of the medications, or by the non-justifiable prescription of non-standard medicines in the institution. The lack of information on the time length of the infusion on the prescription was the most common error involving vancomycin, an important problem because rapid infusions in less than 60 min can lead to macular or maculopapular skin rashes (red man syndrome). ${ }^{33}$

This study has some limitations. The data were obtained from a single institution, which may somehow impair the generalisation of our findings. Furthermore, as DRPs were identified from patient records and medical reports, it is possible that administration errors have been underestimated by recording failures. However, the same methodology has been adopted by other studies in DRP and, considering the scarcity of papers related to the topic of risk factors for DRP in NICU patients, we believe that our results are relevant. The large cohort size, the prospective data collection, the longitudinal design, the in situ evaluation of DRP and the adoption of a well-known DRP classification system are methodological features that contribute to the validity of our results. To our knowledge, this is the first study identifying patient variables and drugs associated with the occurrence of DRP, exclusively in NICU patients. The detection of those predictors is of great value for the identification of patients more prone to DRP, and therefore for the development of screening tools. Such tools can support the work of the healthcare team, especially the clinical pharmacist, with the strengthening of preventive strategies and the optimisation of resources and time.

Further research is needed in order to deepen the study of factors associated with DRP, aiming at the elaboration of risk stratification tools. Future studies should also analyse the influence of external factors on the incidence of DRP, which has not been addressed in our study, such as the number and characteristics of the NICU team members, the workplace conditions, the intrateam and interteam communication, and the organisation of the hospital. Another issue of considerable importance would be the investigation of clinical outcomes of DRP in NICUs.

\section{CONCLUSION}

In conclusion, low gestational age, low 5 min Apgar, neurological disorder, renal disorder and cardiac disorder are risk factors associated with the occurrence of DRP. We also list nine medications with a risk for DRP above the average risk of other medications: alprostadil, amikacin, amphotericin B, cefepime, ciprofloxacin, gentamicin, meropenem, omeprazole and vancomycin. Although they are the most involved in DRP, these medicines account for less than one-third of the drugs prescribed in NICU. Inappropriate dose selection and inappropriate drug use (mainly potential drug incompatibilities) were the main causes of DRP related to those medicines.

Acknowledgements We are grateful to all pharmacists of the maternity hospital, especially to pharmacists Dr Elaine Alves and Dr Tayne Cortez for contributing to the elaboration of the research project, pharmacy residents Kadine Pontes and Bruna Nunes for making available the records of pharmacotherapeutic follow-up of patients, and pharmacy students Mayara Alves and Amanda Nascimento for helping in data collection and tabulation. We also thank all members of the NICU, physicians, physiotherapists, nurses and auxiliaries.

Contributors RDL participated in all stages of the study. MS and TC participated in the study design and revision of the manuscript. RM and $\mathrm{AO}$ contributed to the design and analysis of the study and to the writing and revision of the manuscript. All authors approved the final version of the manuscript.

Funding This study received funding from the National Council for Scientific and Technological Development (CNPq).

Competing interests None declared.

Patient consent for publication Not required.

Ethics approval This study was approved by the Institutional Review Board of the University Hospital Onofre Lopes (No 580.201/2014), which waived the need for written informed consent because the study only evaluated data collected from clinical pharmacy records.

Provenance and peer review Not commissioned; externally peer reviewed. 
Data sharing statement All data generated or analysed during this study are included in this published article. Additional information may be requested directly from the study authors.

Open access This is an open access article distributed in accordance with the Creative Commons Attribution Non Commercial (CC BY-NC 4.0) license, which permits others to distribute, remix, adapt, build upon this work non-commercially, and license their derivative works on different terms, provided the original work is properly cited, appropriate credit is given, any changes made indicated, and the use is non-commercial. See: http://creativecommons.org/licenses/by-nc/4.0/.

\section{REFERENCES}

1. Raju TN, Suresh G, Higgins RD. Patient safety in the context of neonatal intensive care: research and educational opportunities. Pediatr Res 2011;70:109-15.

2. Sorrentino E, Alegiani C. Medication errors in the neonate. The Journal of Maternal-Fetal \& Neonatal Medicine 2012;25:83-5.

3. Chappell K, Newman C. Potential tenfold drug overdoses on a neonatal unit. Arch Dis Child Fetal Neonatal Ed 2004;89:F483-F484.

4. Pharmaceutical Care Network Europe (PCNE). The PCNE Classification V 6.2. 2010 http://www.pcne.org/upload/files/11_ PCNE_classification_V6-2.pdf/ (accessed 15 Apr 2018).

5. Bates DW, Cullen DJ, Laird N, et al. Incidence of adverse drug events and potential adverse drug events. Implications for prevention. ADE Prevention Study Group. JAMA 1995;274:29-34.

6. Kaushal R, Bates DW, Landrigan C, et al. Medication errors and adverse drug events in pediatric inpatients. JAMA 2001;285:2114-20.

7. van den Bemt PMLA, Egberts TCG, de Jong-van den Berg LT, et al. Drug-Related problems in hospitalised patients. Drug Saf 2000;22:321-33.

8. Rashed AN, Neubert A, Tomlin S, et al. Epidemiology and potential associated risk factors of drug-related problems in hospitalised children in the United Kingdom and Saudi Arabia. Eur J Clin Pharmacol 2012;68:1657-66.

9. Allegaert $\mathrm{K}$, van den Anker JN. Adverse drug reactions in neonates and infants: a population-tailored approach is needed. $\mathrm{Br} \mathrm{J}$ Clin Pharmacol 2015;80:788-95.

10. Fernandez-Llamazares CM, Calleja-Hernández MÁ, ManriqueRodríguez S, et al. Prescribing errors intercepted by clinical pharmacists in paediatrics and obstetrics in a tertiary hospital in Spain. Eur J Clin Pharmacol 2012;68:1339-45.

11. van den Anker JN. How to optimize the evaluation and use of antibiotics in neonates. Early Hum Dev 2014;90 Suppl 1:S10-S12.

12. Rodieux F, Wilbaux M, van den Anker JN, et al. Effect of Kidney Function on Drug Kinetics and Dosing in Neonates, Infants, and Children. Clin Pharmacokinet 2015;54:1183-204.

13. Kunac DL, Kennedy J, Austin N, et al. Incidence, preventability, and impact of Adverse Drug Events (ADEs) and potential ADEs in hospitalized children in New Zealand: a prospective observational cohort study. Paediatr Drugs 2009;11:153-60.

14. Sakuma M, Ida H, Nakamura T, et al. Adverse drug events and medication errors in Japanese paediatric inpatients: a retrospective cohort study. BMJ Qual Saf 2014;23:830-7.
15. Ohta Y, Sakuma M, Koike K, et al. Influence of adverse drug events on morbidity and mortality in intensive care units: the JADE study. Int J Qual Health Care 2014;26:573-8.

16. Urbina O, Ferrández O, Grau S, et al. Design of a score to identify hospitalized patients at risk of drug-related problems. Pharmacoepidemiol Drug Saf 2014;23:923-32.

17. Apgar V. A proposal for a new method of evaluation of the newborn infant. Curr Res Anesth Analg 1953;32:260-7.

18. Chow S, Shao J, Wang H. Sample Size Calculations in Clinical Research. 2nd Ed. Boca Raton, FL: Chapman2008.

19. Birarra MK, Heye TB, Shibeshi W. Assessment of drug-related problems in pediatric ward of Zewditu Memorial Referral Hospital, Addis Ababa, Ethiopia. Int J Clin Pharm 2017;39:1039-46.

20. Khdour MR, Jarab AS, Adas HO, et al. Identification of drug-related problems: a prospective study in two general hospitals. Curr Clin Pharmacol 2012;7:276-81.

21. Peterson C, Gustafsson M. Characterisation of Drug-Related Problems and Associated Factors at a Clinical Pharmacist ServiceNaïve Hospital in Northern Sweden. Drugs Real World Outcomes 2017;4:97-107.

22. Rashed AN, Wilton L, Lo CC, et al. Epidemiology and potential risk factors of drug-related problems in Hong Kong paediatric wards. $\mathrm{Br}$ $J$ Clin Pharmacol 2014;77:873-9.

23. Blix HS, Viktil KK, Reikvam A, et al. The majority of hospitalised patients have drug-related problems: results from a prospective study in general hospitals. Eur J Clin Pharmacol 2004;60:651-8.

24. American Academy of Pediatrics, Committee on Fetus and NewbornAmerican College of Obstetricians and Gynecologists and Committee on Obstetric Practice. The Apgar score. Pediatrics 2006;117:1444-7.

25. Pawluk S, Jaam M, Hazi F, et al. A description of medication errors reported by pharmacists in a neonatal intensive care unit. Int $J$ Clin Pharm 2017;39:88-94.

26. Stavroudis TA, Shore AD, Morlock L, et al. NICU medication errors: identifying a risk profile for medication errors in the neonatal intensive care unit. J Perinatol 2010;30:459-68.

27. Wilbaux M, Fuchs A, Samardzic J, et al. Pharmacometric approaches to personalize use of primarily renally eliminated antibiotics in preterm and term neonates. $J$ Clin Pharmacol 2016;56:909-35.

28. Sherwin CM, Medlicott NJ, Reith DM, et al. Intravenous drug delivery in neonates: lessons learnt. Arch Dis Child 2014;99:590-4.

29. Bradley JS, Wassel RT, Lee L, et al. Intravenous ceftriaxone and calcium in the neonate: assessing the risk for cardiopulmonary adverse events. Pediatrics 2009;123:e609-13.

30. Kanji S, Lam J, Johanson C, et al. Systematic review of physical and chemical compatibility of commonly used medications administered by continuous infusion in intensive care units. Crit Care Med 2010;38:1890-8.

31. Foinard A, Décaudin B, Barthélémy C, et al. Impact of physical incompatibility on drug mass flow rates: example of furosemidemidazolam incompatibility. Ann Intensive Care 2012;2:28.

32. Lewis AB, Freed MD, Heymann MA, et al. Side effects of therapy with prostaglandin $\mathrm{E} 1$ in infants with critical congenital heart disease. Circulation 1981;64:893-8.

33. Levy M, Koren G, Dupuis L, et al. Vancomycin-induced red man syndrome. Pediatrics 1990;86:572-80. 